\title{
Attitude of the surgical approach in hyperparathyroidism: A retrospective study
}

\author{
CLAUDIU EDUARD NISTOR ${ }^{1}$, CAMELIA STANCIU-GĂ VAN ${ }^{1}$, FLORINA VASILESCU ${ }^{2}$, \\ ADRIAN VASILE DUMITRU ${ }^{3}$ and ADRIAN CIUCHE ${ }^{1}$ \\ Departments of ${ }^{1}$ Thoracic Surgery and ${ }^{2}$ Pathological Anatomy, \\ 'Dr. Carol Davila' Central Military Emergency University Hospital, 010825 Bucharest; \\ ${ }^{3}$ Department of Pathological Anatomy, Emergency University Hospital Bucharest, 050098 Bucharest, Romania
}

Received April 9, 2021; Accepted May 10, 2021

DOI: $10.3892 /$ etm.2021.10391

\begin{abstract}
The present study constitutes a retrospective study for patients with hyperparathyroidism surgically operated on at the Department of Thoracic Surgery of the Central Military Emergency University Hospital 'Dr. Carol Davila', Bucharest, Romania (SUUMC), over a period of 6 years. The study aimed to elucidate the diagnostic and surgical attitude for an effective treatment, practiced at SUUMC, Romania. The study group included 55 patients: 41 women and 14 men, diagnosed at the endocrinology department, who underwent various personalized surgeries (Kocher modified incision) for typical and ectopic locations of parathyroid pseudotumor formations (hyperplasia and parathyroid adenoma), to cure the disease. The recommended protocol was followed by immediate and 30-day postoperative evaluation which showed normalization of the blood tests, and improved clinical and imaging anomalies. In conclusion, the thoracic surgeon has the necessary knowledge to perform surgery at the cervical, thoracic-cervical and mediastinal levels. Postoperative, the results of laboratory tests for calcium $(\mathrm{Ca})$ and parathyroid hormone (PTH) gradually returned to normal, as can be seen from the statistical study.
\end{abstract}

\section{Introduction}

Diagnosing patients with primary hyperparathyroidism is a challenge. They are often clinically asymptomatic with various biochemical changes in serum levels for calcium $(\mathrm{Ca})$, parathyroid hormone $(\mathrm{PTH})$, vitamin $\mathrm{D}$, and urinary $\mathrm{Ca}(1)$. Increased serum levels of $\mathrm{PTH}$ and $\mathrm{Ca}$ in primary hyperparathyroidism

Correspondence to: Dr Claudiu Eduard Nistor, Department of Thoracic Surgery, 'Dr. Carol Davila' Central Military Emergency University Hospital, 134 Calea Plevnei, Sector 1, 010825 Bucharest, Romania

E-mail: ncd5879@gmail.com

Key words: hyperparathyroidism, hyperplasia, parathyroid adenoma, surgical treatment, Kocher modified incision may be associated with clinical manifestations such as osteoporosis, bone demineralization, nephrolithiasis, dehydration, hypertension, gastrointestinal manifestations (peptic ulcer, pancreatitis) and may even be associated with neuropsychiatric diseases $(1,2)$. Single or multiple adenomas or multiglandular hyperplasia or parathyroid carcinoma can be found in the parathyroid gland. Hyperparathyroidism can be associated with multiple endocrine tumors (genetic syndromes MEN 1 and type 2a) (2). The literature states that asymptomatic hyperparathyroidism has been the most common clinical phenotype in the last 40 years (3).

The etiopathogenesis of hyperparathyroidism is defined by the histopathological diagnosis of surgical resection tissues (adenomas, hyperplasia, cancer) (4). There is also a lack of evidence for personalized molecular diagnosis $(4,5)$, as well as a lack of details on surgical means for a complete resection of pseudotumor and/or parathyroid formations $(6,7)$.

Surgical interventions for pseudotumor or tumor parathyroid formation removal are adapted depending on the anatomical localization of the parathyroid glands (classical or ectopic): classic or minimally invasive cervical parathyroidectomy and mediastinal parathyroidectomy by classical approach (cervicosternotomy), or by mediastinoscopy (6). Guided parathyroidectomy by ultrasound imaging, jugular venous sampling-intraoperative PTH dosing, probe localization, and frozen sections are well known standard procedures (1). These intraoperative adjuvant techniques confirm the efficient removal of the pathological glands $(8,9)$. When parathyroid cancer is suspected, resection is performed with the parathyroid capsule, which is the standard of operation (10).

The complete removal of the parathyroid pseudotumors regardless of localization and anatomical relationships, with decompression of adjacent anatomical formations, requires different surgical approaches. Partial cervicosternotomy is required for mediastinal parathyroid large formations or fixated to the anatomical structures of the mediastinum (11-13). In some cases, we used modified classical cervicotomy and modified Kocher cervicotomy $(14,15)$.

We conducted this retrospective study to reveal the diagnostic attitude and surgical approaches applied by our team; the advantages of Kocher modified incision. We consulted international protocols for hyperparathyroidism in pseudotumor 
lesions: hyperplasias or parathyroid adenomas. We underline the role of the thoracic surgeon for the surgical interventions of these formations with the typical and ectopic location.

\section{Patients and methods}

This study was reported according to STROBE (Strengthening the Reporting of Observational Studies in Epidemiology) guidelines (16). This is a retrospective study. The study was approved by the Research Ethics Committee carried out through the collaboration of the 'Carol Davila' University of Medicine and Pharmacy from Bucharest and the Central Military Emergency University Hospital 'Dr. Carol Davila' Bucharest (no. 388/09 June 2020). We retrospectively studied the records of the patients who underwent surgery against pseudotumor formations with hyperparathyroidism in SUUMC, between 2014 and 2019. The interventions were performed in a single surgical center.

Participants. With these guides as a benchmark, we analyzed the surgical treatment of hyperparathyroidism and the inclusion criteria for surgery (increased values of serum Ca; levels of PTH compared to reference values; biochemical analysis of renal function; the presence of renal lithiasis; the presence of osteoporosis on X-rays; the age of each patient). We evaluated the therapeutic efficacy by comparing preoperative to postoperative values. A database of clinical and paraclinical signs was created from observation sheets, operatory protocols, and histopathological reports.

The study group comprised 41 women and 14 men with a mean age of 54 years and ages between 21 and 87 years. Initially, the patients were selected by the endocrinologist based on clinical diagnosis, laboratory, and imaging criteria. Blood samples for serum Ca and PTH were analyzed for all patients. A total of 51 patients were diagnosed with primary hyperparathyroidism, 3 patients were diagnosed with secondary hyperparathyroidism and one patient was diagnosed with tertiary hyperparathyroidism.

The selection of patients for surgical interventions was made based on international protocols for diagnostic management.

Clinical analysis. Clinical analysis included: fatigue in 42 patients, bone pain in 49 patients, myalgia in 36 patients, polyuria in 22 patients, polydipsia in 26 patients, renal lithiasis in 37 patients, hypertension in 32 patients, osteoporosis in 48 patients, and depression in 34 patients.

Laboratory analysis. Preoperative and postoperative blood samples were analyzed immediately after collection. Preoperative blood sample investigations in patients detected serum Ca levels $>8.40-10.2 \mathrm{mg} / \mathrm{dl}$ (considered to be normal in the reference values). A total of 51 patients presented equivalent values for primary hyperparathyroidism, 3 secondary and one tertiary; an increased serum level of PTH $>15-65 \mathrm{pg} / \mathrm{ml}$ (considered to be the normal reference range) was detected in all patients in the group and an increased serum level of total proteins $>6.5-8.1 \mathrm{~g} / \mathrm{dl}$ (normal reference range) was also detected in all patients. Postoperative serum levels of these analytes were normal or close to normal values.
Imaging diagnosis. All patients underwent an X-ray for bone conditions (48 patients had osteoporosis), and ultrasound evaluations of soft parts of the cervical region were performed for all patients. Renal ultrasounds were performed for all patients (37 patients were diagnosed with renal lithiasis), 99mTc-sestamibi scintigraphy, and computed tomography (CT). Patients who received intraoperative ultrasound cervical examination aided the surgical team approach to target the pseudotumor formation.

Histopathological diagnosis. The confirmation of the diagnosis of the excised parts was established by the pathological anatomy service. Twelve specimens with parathyroid hyperplasia were found and 43 were parathyroid adenomas.

The therapeutic attitude complied with the international treatment management protocols for hyperparathyroidism (11). Thus, depending on the typical anatomical (cervical) or ectopic (intramediastinal) localization of the pseudotumor formations, 36 parathyroidectomies surgical approaches through minimal incisions centered on formations under ultrasound guidance, 11 classical parathyroidectomies, 3 partial cervical sternotomies and 8 excisions by mediastinoscopy were performed. A total of 6 patients benefited from autotransplantation of a parathyroid gland, in the deltoid muscle or the sternocleidomastoid muscles.

\section{Results}

High therapeutic efficacy is achieved by curing the hyperparathyroidism, decreasing morbidities related to the disease, increasing the quality of life of the patient, decreasing hospitalization days for severe correction of hypercalcemia or acute renal failure, and reducing direct costs of treatment and hospitalization time.

Characteristics of the patients. The endocrinologist selected the patients with hyperparathyroidism who required surgery. Only patients who had undergone surgery were studied. The symptomatology was specific for hyperparathyroidism: fatigue in 42 patients, bone pain in 49 patients, myalgia in 36 patients, polyuria in 22 patients, polydipsia in 26 patients, renal lithiasis in 37 patients, hypertension in 32 patients, osteoporosis in 48 patients, and depression in 34 patients. Examinations of the blood for calcemia, PTH, and proteinemia were carried out preoperatively as well as postoperatively. Preoperative PTH values 73 and $210 \mathrm{pg} / \mathrm{ml}$ decreased after surgery to $48-65 \mathrm{pg} / \mathrm{ml}$.

Localization of the tumors. Imaging investigations established the following localizations of the pseudotumoral parathyroid formations: Right upper parathyroid in 6 cases, right lower parathyroid in 26 cases, left upper parathyroid in 7 cases, left lower parathyroid in 10 cases, right retrothyroidian parathyroid in 3 cases, left retrothyroidian parathyroid in 5 cases, right paratracheal mediastinal parathyroid in 3 cases, parathyroid in the anterior mediastinum in 5 cases, right mediastinal cervical parathyroid in 4 cases, parathyroid adherence of the brachiocephalic artery trunk in 1 case. It should be mentioned that 43 patients had a single pseudotumoral formation of parathyroid disease, 9 patients were detected with two formations, and 3 patients with 3 formations. 
After the classification of Perrier parathyroid adenomas, the locations of the pseudotumors were: type A in 24 formations, type $\mathrm{B}$ in 11 formations, type $\mathrm{C}$ in 13 cases, type $\mathrm{D}$ in 3 cases, type $\mathrm{E}$ in no cases, type $\mathrm{F}$ in 14 cases, and type $\mathrm{G}$ in 5 cases.

Surgical approach. Typical or ectopic anatomical localizations indicated the type of surgical approach. We performed classical parathyroidectomy for bilateral or multiple parathyroid lesions (classic Kocher incision), parathyroidectomy through Kocher modified incision (for the superior parathyroid lesions), minimally invasive parathyroidectomy (for the inferior parathyroid lesions), and mediastinal parathyroidectomy (through upper cervicosternotomy or by mediastinoscopy). We also performed 6 associated parathyroid autotransplantations in some cases. For successful operative management, parathyroid pseudotumoral formations were spotted both preoperatively and intraoperatively (38 formations) by ultrasound.

For patients who had upper right parathyroid pseudotumoral formations ( 6 cases) and for 7 cases of upper left localized parathyroid lesions, a modified Kocher incision was performed, approximately $3 \mathrm{~cm}$ in length, to allow for an efficient surgical approach of the area containing the lesion. After a thorough dissection of the anatomical planes, with the identification of the ipsilateral recurrent laryngeal nerve and its preservation, as well as the control of the upper thyroid artery, the posterior face of the thyroid gland was reached where the pseudotumoral formations were found. Aspects of the parathyroid pseudotumoral formations included: A yellowish-brown color, encapsulated, and the vast majority were relatively easily delineated from the tissue of the thyroid gland, but 4 formations were difficult to delineate from the adjacent thyroidal peri-glandular tissues, with diameters between 1 and $2 \mathrm{~cm}$. No partial or total thyroidectomy was required in any patient.

There were 26 cases of lower right parathyroid pseudotumoral formations, and 10 cases of lower left parathyroid gland, for which incisions centered on the pseudotumoral formation were made. Preoperatively, the tegument was marked under ultrasound guidance, then a minimal incision was made in the marked area. In the dissection of the anatomical layers, special attention was paid to the inferior thyroid arteries and the ipsilateral recurrent laryngeal nerve respectively, but some 4 formations were difficult to delineate from the thyroid peri-glandular tissues.

Modified Kocher type incision was carried out in the lower right retro-thyroid pseudotumoral formations (3 cases) and for the lower-left one (1 case). Increased attention was paid to vasculonervous formations, especially the recurrent laryngeal nerve, upper and lower thyroid arterial branches.

Carlen's type cervical mediastinoscopy was performed in 8 cases of mediastinal parathyroid pseudotumoral formations. After the incision, the cervical anatomical planes were carefully dissected and soft dissection was performed at the level of the upper mediastinum, below the arterial vascular plane represented by the brachiocephalic artery trunk and aorta. Paratracheal pseudotumoral formations were removed under the mediastinoscopy, 3 cases from the right (adjacent to the remaining thymic tissue), and 5 cases from the left (in the mediastinal fat).

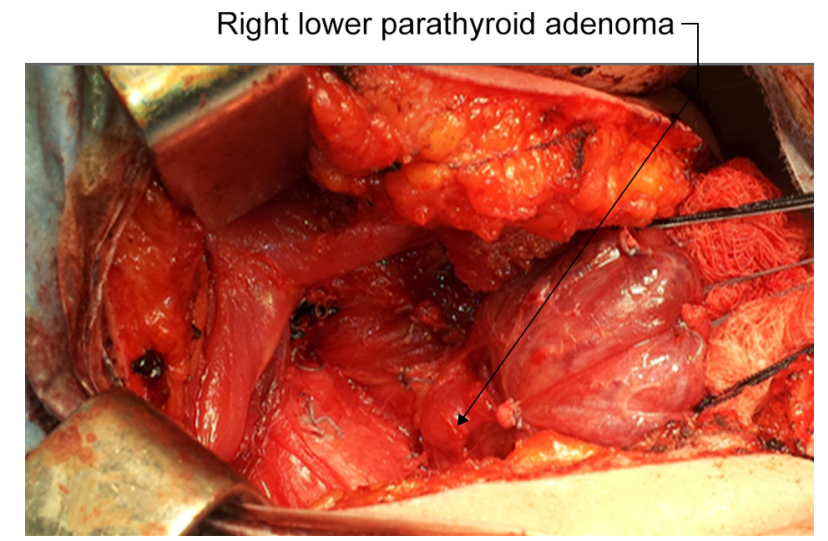

Figure 1. Right lower parathyroid adenoma.

A minimal suprasternal cervicotomy was performed in 1 case of parathyroid adenoma adherent to the brachiocephalic artery trunk. After dissection of the anatomical layers, a pseudotumoral formation with a diameter of $2.5 \mathrm{~cm}$ was found, located at the level of the brachiocephalic artery trunk. In this case, the experience of the operative team was necessary to perform an excision of this pathological formation without complications. A total of 6 patients required parathyroid autotransplantation. In this context, parathyroid pseudotumoral (hyperplasia) formation was detected. Excision of the gland was performed, 1/3 was sent for histopathological examination and 2/3 was re-implanted at the level of the right deltoid muscle and sternocleidomastoid muscles. Also in these cases, the experience of the surgical team was necessary for this attitude of preservation of the parathyroid tissue, aimed to maintain the parathyroid function (Fig. 1).

Partially parathyroid autotransplantation in the deltoid muscle after parathyroidectomy was made for PTH level adjustment. Tissue samples were subsequently sent for evaluation to the pathology department of the same hospital. The specimen samples were fixated with $10 \%$ buffered formalin and were processed by conventional histopathological methods using paraffin-embedding, 2-micron sectioning, and hematoxylin and eosin (H\&E) staining. Histopathological examination of the standard $\mathrm{H} \& \mathrm{E}$-stained slides revealed in most cases typical forms of parathyroid adenomas and a few cases of parathyroid hyperplasia. The tumoral masses were composed of chief cells, oncocytes (including transitional oncocytes), or an admixture of these cell types. Pathological anatomy examinations were analyzed for all of the surgically operated patients. In 6 cases $(11 \%)$ parathyroid hyperplasias were found and in 49 cases (89\%) parathyroid adenomas.

Primary result. The results of the laboratory analyses carried out immediately postoperatively showed values close to the reference ranges considered normal. No expanding hematoma was found in the pretracheal space in any patient, neither laryngeal edema nor dysphonia or phony.

Secondary results. At the check-up carried out 30 days after surgery, all patients presented normal values of the reference serological analyses provided in the protocols. 


\section{Discussion}

Surgical treatment is necessary in the cases of primary hyperparathyroidism and parathyroidectomy as a therapeutic strategy is realized through numerous approaches $(1,8,17)$. The thoracic surgeon plays a very important role in the application of the surgical treatment. The approaches for parathyroid surgery are similar to the approaches for the thyroid (18-20). Typical and ectopic anatomical localization of the pathological parathyroid glands requires an experienced team in the surgery of parathyroid and thyroid glands $(21,22)$. These localizations may often require surgery performed at the anatomical border between the cervical and thoracic regions, requiring several surgical specialties (otorhinolaryngology, oromaxillofacial surgery, thoracic surgery). The localization of these pseudotumoral formations at the level of the lower neck, upper thorax, anterior mediastinum, paratracheal mediastinal region, requires the experience of a thoracic surgeon (23). Our team of surgeons has developed modified surgical approaches to the classical ones, as follows.

The modified Kocher cervical incision was used in the situation of preoperative suspicion of parathyroid glandular hyperplasia as a cause of primary hyperparathyroidism. This incision, which the medical literature (24-26) does not mention as such, consists of a suprasternal, symmetric cervical incision, up to $3 \mathrm{~cm}$ long, centered on the midline of the neck. We have successfully used it in surgeries performed for parathyroid glandular hyperplasia because it has some advantages over standard Kocher cervicotomy. Thus, in addition to the aesthetic advantage, this incision allows better visualization and dissection of the posterior face of the thyroid lobes to identify the parathyroid gland quite difficult to observe and isolate in rich perithyroidal fat. It also allows the bilateral approach of the parathyroid glands. This incision is more important as we are in the situation of lower parathyroid glandular hyperplasia. The approach of the upper parathyroid hyperplasia was more difficult to perform on this path. Another advantage of this incision is the possibility to extend it bilaterally in the shape of the classical Kocher cervical incision, in the situation required by the too-high localization of glandular hyperplasia and in the case of hyperplastic parathyroid glands inclavated intrathyroidal, which may require the bulk removal of the parathyroid gland and the thyroid lobe.

Mediastinoscopy allows easier visual identification (by magnifying the video image) (27-29) of parathyroid adenomas localized either peritracheal and retro-vascular or at the level of the anterosuperior mediastinum and permitted complete removal of parathyroid ectopic tumor formations (30-32).

For the patients with a positive diagnosis of hyperparathyroidism, we made a personalized surgical approach, with a histopathological profile of excised parathyroid formations, followed by endocrinological reassessment. The other patients were referred to the endocrinology service.

In conclusion, the attitude of the diagnosis and the surgical approach in hyperparathyroidism made by our team resulted in the cure of patients with hyperparathyroidism associated with parathyroid hyperplasia and parathyroid adenoma. The surgical approaches aimed at total excision of the pathological formations with minimal lesions, rapid healing, decrease in the number of days of hospitalization, and early social reintegration.

\section{Acknowledgements}

Many thanks to 'Dr. Carol Davila' Central Military Emergency University Hospital Bucharest, Romania and 'Carol Davila' University of Medicine and Pharmacy Bucharest for supporting our study.

\section{Funding}

No funding was received.

\section{Availability of data and materials}

The data that support the findings of this study are available from the corresponding author (CEN), upon reasonable request.

\section{Authors' contributions}

CEN and AC recruited and carried out the patient surgery. CSG was part of the operating team and performed data collection and statistical analysis. FV and AVD processed the biological samples collected by $\mathrm{CEN}$ and AC performed the anatomopathological examination. All authors designed, read and agreed to the published version of the manuscript.

\section{Ethics approval and consent to participate}

The study was approved by the Research Ethics Commission from 'Dr. Carol Davila' Central Military Emergency University Hospital Bucharest (no. 388/3.06.2020).

\section{Patient consent for publication}

Not applicable.

\section{Competing interests}

The authors declare that they have no competing interests.

\section{References}

1. Parnell KE and Oltmann SC: The surgical management of primary hyperparathyroidism: An updated review. Int J Endocr Oncol 5: IJE07, 2018.

2. DeLellis RA, Mazzaglia P and Mangray S: Primary hyperparathyroidism: A current perspective. Arch Pathol Lab Med 132: $1251-1262,2008$

3. Silverberg SJ, Clarke BL, Peacock M, Bandeira F, Boutroy S, Cusano NE, Dempster D, Lewiecki EM, Liu JM, Minisola S, et al: Current issues in the presentation of asymptomatic primary hyperparathyroidism: Proceedings of the fourth international workshop. J Clin Endocrinol Metab 99: 3580-3594, 2014.

4. Mizamtsidi M, Nastos C, Mastorakos G, Dina R, Vassiliou I, Gazouli M and Palazzo F: Diagnosis, management, histology and genetics of sporadic primary hyperparathyroidism: Old knowledge with new tricks. Endocr Connect 7: R56-R68, 2018.

5. Mamedova E, Mokrysheva N, Vasilyev E, Petrov V, Pigarova E, Kuznetsov S, Kuznetsov N, Rozhinskaya L, Melnichenko G, Dedov I and Tiulpakov A: Primary hyperparathyroidism in young patients in Russia: High frequency of hyperparathyroidism-jaw tumor syndrome. Endocr Connect 6: 557-565, 2017.

6. Pappachan JM, Sodi R, Viswanath AK and Lahart IM: Parathyroidectomy for adults with primary hyperparathyroidism (Protocol). Cochrane Database Syst Rev 2018: CD013035, 2018. 
7. National Institute for Health and Care Excellence (NICE): Hyperparathyroidism (primary): Diagnosis, assessment and initial management. NICE guideline [NG132]. https://www.nice. org.uk/guidance/ng132/chapter/Recommendations. Accessed May 23, 2019.

8. Udelsman R, Åkerström G,Biagini C, Duh QY, Miccoli P, Niederle B and Tonelli F: The surgical management of asymptomatic primary hyperparathyroidism: Proceedings of the fourth international workshop. J Clin Endocrinol Metab 99: 3595-3606, 2014.

9. Udelsman R, Lin Z and Donovan P: The superiority of minimally invasive parathyroidectomy based on 1650 consecutive patients with primary hyperparathyroidism. Ann Surg 253: 585-591, 2011

10. Wilhelm SM, Wang TS, Rua DT, Lee JA, Asa SL, Duh OY, Doherty GM, Herrera MF, Pasieka JL, Perrier ND, et al: The American association of endocrine surgeons guidelines for definitive management of primary hyperparathyroidism. JAMA Surg 151: 959-968, 2016.

11. Nistor Cl, Ciuche A and Horvat T: Thoracic Surgery. Vol IV. Mediastinal pseudotumors. Treaty of Surgery. 1st edition. Romanian Academy Publishing House, Bucharest, pp701-704, 2008.

12. Spiroiu C, Ranetti AE and Nistor C: Giant parathyroid adenoma with severe hypercalcaemia: Case report. Endocrine Abstracts 37: EP296, 2015

13. Nistor C, Davidescu M, Ciuche A, Rus O, Tudose A, Vasilescu F and Horvat T: Giant multifocal thyroid tumor. Maedica-a Journal of Clinical Medicine 4: 346-352, 2009.

14. Nistor C, Ciuche A, Motas C, Motas N, Bluoss C, Pantile D, Davidescu $\mathrm{M}$ and Horvat T: Cervico-mediastinal thyroid masses-our experience. Chirurgia (Bucur) 109: 34-43, 2014.

15. Nistor C, Ciuche A and Constantinescu I: Emergency surgical tracheal decompression in a huge retrosternal goiter. Acta Endocrinol (Buchar) 13: 370-374, 2017

16. von Elm E, Altman DG, Egger M, Pocock SJ, Gøtzsche PC and Vandenbroucke JP; STROBE Initiative: The Strengthening the reporting of observational studies in epidemiology (STROBE) statement: Guidelines for reporting observational studies. Ann Intern Med 147: 573-577, 2007.

17. Song CM, Ji YB, Kim IS, Lee JY, Kim DS and Tae K: Low transverse incision for lateral neck dissection in patients with papillary thyroid cancer: Improved cosmesis. World J Surg Oncol 15: 97, 2017.

18. Noureldine SI, Gooi Z and Tufano RP: Minimally invasive parathyroid surgery (Review). Gland Surg 4: 410-419, 2015.

19. Henry JF: Minimally invasive thyroid and parathyroid surgery is not a question of length of the incision. Langenbecks Arch Surg 393: 621-626, 2008 .
20. Duke WS and Terris DJ: Alternative approaches to the thyroid gland. Endocrinol Metab Clin North Am 43: 459-474, 2014.

21. Mazeh H, Stoll SJ, Robbins JB, Sippel RS and Chen H: Validation of the 'Perrier' parathyroid adenoma location nomenclature. World J Surg 36: 612-616, 2012.

22. Simo R, Nixon I, Tysome JR, Balfour A and Jeannon JP: Modified extended Kocher incision for total thyroidectomy with lateral compartment neck dissection-a critical appraisal of surgical access and cosmesis in 31 patients. Clin Otolaryngol 37: 395-398, 2012

23. Irvin GL III, Carneiro DM and Solorzano CC: Progress in the operative management of sporadic primary hyperparathyroidism over 34 years. Ann Surg 239: 704-708, discussion 708-711, 2004

24. Runge T, Inglin R, Riss P, Selberherr A, Kaderli RM, Candinas D and Seiler CA: The advantages of extended subplatysmal dissection in thyroid surgery-the 'mobile window' technique. Langenbecks Arch Surg 402: 257-263, 2017.

25. Lin N, Yu L, Ma J and Wang Y: Minimally invasive nonendoscopic thyroidectomy with a low anterior cervical incision. Clin Oncol 6: Article 1776, 2021.

26. Elkanovich T, Hajouj M and Ronen O: The natural neck crease as an anatomic landmark for thyroid surgery incision. Surgeon 30: S1479-S1666, 2020

27. Spear C, Geraci T, Bizekis C and Zervos M: Resection of an ectopic parathyroid adenoma via video-assisted mediastinoscopy. Semin Thorac Cardiovasc Surg 31: 323-325, 2019.

28. Liman ST, Topcu S, Dervisoglu E, Gorur GD, Elicora A, Burc K and Akgul AG: Excision of ectopic mediastinal parathyroid adenoma via parasternal videomediastinoscopy. Ann Thorac Cardiovasc Surg 20: 67-69, 2014.

29. Röösli C, Bortoluzzi L, Linder TE and Müller W: Role of minimal invasive surgery for primary and secondary hyperparathyroidism. Laryngorhinootologie 88: 460-464, 2009 (In German).

30. Razzak R, McMullen T and Bédard ELR: Excision of middle mediastinal parathyroid adenoma by videoscopic assisted mediastinoscopy (VAM). J Thorac Dis 8: 2651-2653, 2016.

31. Tcherveniakov P, Menon A, Milton R, Papagiannopoulos K, Lansdown M and Thorpe JA: Video-assisted mediastinoscopy (VAM) for surgical resection of ectopic parathyroid adenoma. J Cardiothorac Surg 2: 41, 2007.

32. Toktaş O, İliklerden Ü, Yerlikaya B, Kotan Ç and Batur A: Transcervical resection of two parathyroid adenomas located on the anterior mediastinum. Turk J Surg 34: 247-249, 2018. 